\title{
PROOF OF THE GELFAND-KIRILLOV CONJECTURE FOR SOLVABLE LIE ALGEBRAS
}

\author{
A. JOSEPH
}

ABSTRACT. Let $g$ be a solvable algebraic Lie algebra over the complex numbers $C$. It is shown that the quotient field of the enveloping algebra of $g$ is isomorphic to one of the standard fields $D_{n, k}$, being defined as the quotient field of the. Weyl al gebra of degree $n$ over $C$ extended by $k$ indeterminates. This proves the Gelfand-Kirillov conjecture for $g$ solvable.

1. Introduction. Let $g$ be an algebraic Lie algebra over the complex numbers C. Let $U g$ denote the enveloping algebra of $g$ and $D g$ the quotient field of $U g$. With $n, k$ nonnegative integers, let $A_{n, k}$ denote the Weyl algebra of degree $n$ over $\mathbf{C}$ extended by $k$ indeterminates [1]. 'Let $D_{n, k}$ denote the quotient field of $A_{n, k}$. It has been conjectured [1] $-[3]$ that $D g$ is isomorphic to $D_{n, k}$ for suitable $n, k$. This has been demonstrated for $g$ nilpotent and for $g$ semisimple if either $g=s l(r)$ [2] or $D g$ is given an extended centre [3]. Here we prove the conjecture for $g$ solvable and algebraic. The analysis is based on the work of Nghiêm [4] and the theorem proved below.

2. A transcendence theorem. Let $g$ be a finite dimensional Lie algebra over $\mathrm{C}$ and $A$ an arbitrary commutative subalgebra of $U g$. Let $\operatorname{Dim}_{C}$ denote the dimensionality introduced by Gelfand and Kirillov [1]. We have shown [5, Theorem 1.1] that

$$
\operatorname{Dim}_{C} A \leq \operatorname{dim} g-1 / 2 \operatorname{dim} \Omega,
$$

where $\Omega$ is an orbit of maximal dimension in the dual $g^{*}$ of $g$ [1].

Recall that if $g$ is algebraic, then in particular $g \subset g l(V)$ for some finite dimensional vector space $V$ over C. $g \subset g l(V)$ is said to be almost algebraic [6, p. 98] given that the semisimple and nilpotent parts of every

Received by the editors April 12, 1973.

AMS (MOS) subject classifications (1970). Primary 17B35; Secondary 16 A08.

Key words and phrases. Gelfand-Kirillov conjecture, quotient field of Lie algebra, Weyl algebra. 
$X \in g$ belong to $g . g$ is almost algebraic if it is algebraic [7, Lemma 2, \$3]. The proofs of the following two lemmas are straightforward (cf. [8, in particular, Theorem 4]).

Lemma 2.1. Let $g \subset g l(V)$. Given $X \in g$ semisimple (resp. nilpotent) then $\operatorname{ad}_{g} X$ is semisimple (resp. nilpotent).

Lemma 2.2. Let $g$ be solvable and almost algebraic. Then $g=g_{1} \oplus$ $g_{2}$ where $g_{1}$ (resp. $g_{2}$ ) is the commutative subalgebra (resp. nilpotent ideal) of $g$ spanned by the semisimple (resp. nilpotent) elements of $g$.

Proof. Let $g_{1}$ (resp. $g_{2}$ ) denote a maximal abelian subalgebra of semisimple (resp. the set of all nilpotent) elements of $g$. Certainly $g_{1} \cap g_{2}=$ $\{0\}$. By Lie's theorem, it follows that $g_{2}$ is a linear space and $g_{2} \supset[g, g]$, so $g_{2}$ is an ideal. Set $a=g_{1} \oplus g_{2} \cdot a$ is an invariant subspace of ad $g_{1}$, so by Lemma 2.1 and the choice of $g_{1}, a$ is complemented in $g$. That is $g=a \oplus b$, with $\left[g_{1}, b\right] \subset b$. Yet $b \cap g_{2}=\{0\}$, so $\left[g_{1}, b\right]=\{0\}$. Since $g$ is almost algebraic, we may write for each $X \in b: X=Y+Z ; Y, Z \in g$, where $Y, Z$ are the semisimple and nilpotent parts of $X$. We have $\left[g_{1}, Y\right]=\{0\}$, so $Y=0$, by the maximality of $g_{1}$. Then $Z=0$, since $b \cap g_{2}=\{0\}$. Hence $b=\{0\}$, and $a=g$ as required.

Given $g \subset g l(V)$, let $S(V)$ denote the symmetric algebra over $V$ and $K(V)$ its quotient field. Define the action of $g$ on $S(V)$ by derivation and on $K(V)$ by the rule $X \xi=-a^{-2}(X a) b+a^{-1}(X b)$ where $\xi=a^{-1} b \in K(V), a, b \in$ $S(V)$. More generally we wish to consider the possibility of there being certain additional algebraic relations in $S(V)$. Let $I$ denote the (two-sided) ideal generated by finitely many $g$ annulled elements of $K(V)$. Assume $I$ prime. Set $S=S(V) / I$ and $K$ its quotient field. Define the subfield $K_{0}$ of $K$ annihilated by $g$ through

$$
K_{0}=\{\xi \in K: X \xi=0, \text { for all } X \in g\} .
$$

Let deg $K$ denote the degree of transcendence of $K$.

Theorem 2.3. Suppose $g \subset g l(V)$ is solvable and almost algebraic. Define $K_{0} K_{0}$ as above. Then $K$ is a pure transcendental extension of $K_{0}$. Further, given $g$ nilpotent, $\operatorname{deg} K-\operatorname{deg} K_{0} \leq \operatorname{dim} g$.

Proof. Let $g=g_{1} \oplus g_{2}$ be the decomposition of $g$ defined in the conclusion of Lemma 2.2. Set $T=\left\{a \in S: g_{2} a=0\right\}$. Then $g T \subset T$, since $g_{2}$ is an ideal. Let $L$ denote the quotient field of $T$ and $L_{0}$ the subfield of 
$L$ annihilated by $g_{1}$. Then $K_{0}=L_{0}$. Indeed $K_{0} \supset L_{0}$ is immediate. On the other hand given $\xi \in K_{0}$, write $\xi=a^{-1} b: a, b \in S$. Suppose that there exists a $Z \in g_{2}$ such that $Z a \neq 0$. Since $Z \xi=0$, we obtain $\xi=(Z a)^{-1} Z b$. Since $g_{2}$ is nilpotent it follows that we may write $\xi=c^{-1} d: c, d \in T$. Hence $K_{0} \subset L_{0}$ and so $K_{0}=L_{0}$ as required.

Next show that $L$ is a pure transcendental extension of $L_{0}$. Let $S^{i} \subset S$ denote the linear subspace of homogeneous polynomials of degree $i$. Set $T^{i}=T \cap S^{i}$. For each $i, S^{i}$ and $T^{i}$ are finite dimensional, $g$ invariant and respectively define a direct sum decomposition of $S$ and $T$.

Set $\operatorname{dim} g_{1}=r, \operatorname{dim} g_{2}=s$. Let $\left\{Y_{i}: i=1,2, \cdots, r\right\}$ be a basis for $g_{1}$. Let $\operatorname{spec}(S)$ (resp. spec $(T)$ ) be the set of all $r$-tuples $\lambda$ such that

$$
Y_{i} \xi=\lambda_{i} \xi: \quad i=1,2, \cdots, r,
$$

$\xi \in S(\lambda)$ (resp. $T(\lambda)$ ) where $S(\lambda), T(\lambda)$ denote the corresponding eigensubspaces. These subspaces define a direct sum decomposition of $S$ and $T$ respectively. Define $L(\lambda), \operatorname{spec}(L)$ analogously. Since $I$ is prime a monomial cannot vanish in $S$. Hence $\operatorname{spec}(S)$ and $\operatorname{spec}(T)$ are closed under the addition of $r$-tuples. We remark that $\operatorname{spec}(S) \supset \operatorname{spec}(T)$ and that this may be strict inclusion. Further spec $(S)$ is generated over the integers by $\operatorname{spec}\left(S^{1}\right)$. Let $W$ be the vector space generated by $\operatorname{spec}\left(S^{1}\right)$ over the rationals. Set $t=\operatorname{dim} W \leq \operatorname{dim} V$. Let $\left\{\lambda_{j} \in \operatorname{spec}\left(S^{1}\right)\right\}$ be a basis for $W$. Let $u$ be the common divisor for the rational coefficients of the remaining $\boldsymbol{\mu}_{j} \in \operatorname{spec}\left(S^{1}\right)$ expressed in this basis. Then for all $\lambda \in \operatorname{spec}(T)$ we have

$$
\lambda=\frac{1}{u} \sum_{j=1}^{t} u_{j} \lambda_{j}
$$

for suitable integers $u_{j}$. Let $M$ be the module over the integers generated by $\operatorname{spec}(T)$. With respect to (2.2) define a map $\pi: M \rightarrow Z^{t}$, through $\pi(\lambda)=\mathbf{u}$. Then $M$ can be considered as a submodule of $Z^{t}$. Hence $M$ is a finitely generated free $\mathrm{Z}$ module. Let $\left\{\Lambda_{a}\right\}$ be a basis for $M$. Taking products of elements of $T$ with integer exponents gives $M=\operatorname{spec}(L)$, so there exist $y_{a} \epsilon$ $L(\lambda)$ such that $Y_{i} y_{a}=\Lambda_{i a} y_{a}$ for all $i, a$. Further by definition of $\left\{\Lambda_{a}\right\}$ distinct monomials in the $y_{a}$ belong to distinct eigensubspaces $L(\lambda)$. Hence they are algebraically independent over $L_{0}$. Now given $u, v \in T(\lambda), u v^{-1} \in$ $L_{0}$, so $u=b v$ for some $b \in L_{0}$. Recalling the direct sum decomposition of $T$, it follows that $L+L_{0}\left(y_{1}, y_{2}, \cdots, y_{t}\right): t \leq \operatorname{dim} V$ as required.

Next show that $K$ is a pure transcendental extension of $L$. In this it is convenient to set $g_{1}=h, g_{2}=g$. Since $g$ is nilpotent, there exists an upper 
central series $\{0\}=C_{1} \subset C_{2} \subset C_{3} \subset \cdots \subset C_{l}=g$, for $g$ [6, p.29]. (Recall that $C_{i}$ is the maximal ideal in $g$ such that $\left[g, C_{i}\right] \subset C_{i-1}$.) That $\left[h, C_{i}\right] \subset$ $C_{i}$, follows by induction on $i$ and the relation $\left[g,\left[h, C_{i}\right]\right] \subset C_{i-1}+\left[h, C_{i-1}\right]$. By definition of $h$ and Lemma 2.1, ad ${ }_{g} h$ is a commutative Lie algebra of semisimple elements. Hence there exists a central descending series $g=g_{1} \mathrm{~J}$ $g_{2} \supset \cdots \supset g_{s+1}=\{0\}: s=\operatorname{dim} g$, for $g$ with the property that for each integer $k$ there exists $Z_{k} \in g_{k}, Z_{k} \notin g_{k+1}$ such that $Z_{k}$ is an eigenvector for ad $g_{g}$. (Recall also that $\operatorname{dim} g_{k}=\operatorname{dim} g_{k+1}+1$ and $\left[g, g_{k}\right] \subset g_{k+1}$, for all $k_{\text {.) }}$

Let $L_{k}$ denote the subfield of $K$ annihilated by $g_{k}$. Then $L=L_{1} C$ $L_{2} \subset \cdots \subset L_{s+1}=K$. Let $\tilde{S}$ be the maximal subalgebra of $K \underset{\sim}{\text { on which the }}$ elements of $g$ are locally nilpotent derivations. Set $\tilde{S}_{k}=L_{k} \cap \tilde{S}$ and $S_{k}=$ $L_{k} \cap S$. Certainly $\tilde{S}_{k}$ contains the subalgebra of $K$ generated by $L$ and $S_{k}$. In fact we shall see that it coincides with it.

We show that for each $k$ either $L_{k+1}=L_{k}$ or $L_{k+1}=L_{k}(a)$ for some $a \in \tilde{S}_{k+1}$. This will prove the theorem with $\operatorname{deg} K-\operatorname{deg} L \leq \operatorname{dim} g$ as required. Verify that $Z_{i} S_{j} \subset S_{j}, Z_{i} \tilde{S}_{j} \subset \widetilde{S}_{j}$ and $Z_{i} L_{j} \subset L_{j}$ for all $i, j$. We show that $L_{k}$ is the quotient field of $S_{k}$ by induction on $k$. By definition $K=L_{s+1}$ is the quotient field of $S=S_{s+1}$. So, given $\xi \in L_{k} \subset L_{k+1}$, we may write by the induction hypothesis $\xi=a^{-1} b: a, b \in S_{k+1}$ Suppose that $Z_{k} a \neq 0$, then $Z_{k} \xi=0$ gives $\xi=\left(Z_{k} a\right)^{-1} Z_{k} b$. Then by the local nilpotency of $Z_{k}$ on $S_{k+1}$ we may choose $a, b \in S_{k}$.

Finally suppose that $L_{k+1} \neq L_{k}$. Then there exists $a^{\prime} \in S_{k+1}$ such that $Z_{k} a^{\prime} \neq 0$ so by the local nilpotency of $Z_{k}$ on $S_{k+1}$, there exists $a^{\prime \prime} \in S_{k+1}$ such that $Z_{k} a^{\prime \prime}=b \in S_{k}: b \neq 0$. Now suppose that $Z_{i} b \neq 0$, for some $i$. Then $Z_{k}\left(Z_{i} a^{\prime \prime}\right)=\left[Z_{k}, Z_{i}\right] a^{\prime \prime}+Z_{i}\left(Z_{k} a^{\prime \prime}\right)=Z_{i} b \neq 0$, since $\left[Z_{k}, Z_{i}\right] \in g_{k+1}$ and $a^{\prime \prime} \in S_{k+1}$. Hence by the local nilpotency of $Z_{i}$ on $S_{k}$ we may choose $a^{\prime \prime \prime} \epsilon$ $S_{k+1}$ such that $Z_{k} a^{\prime \prime \prime}=b^{\prime} \in L: b^{\prime} \neq 0$. Then there exists $a \in \widetilde{S}_{k+1}$ such that $Z_{k} a=1$. Let $a_{n} a^{n}+\cdots+\alpha_{0}: a_{i} \in L_{k}, a_{n} \neq 0$, be the minimal polynomial of $a$ in $L_{k}$. The relation $Z_{k} a^{i}=i a^{i-1}$ contradicts its minimality, so $a$ is transcendental over $L_{k}$. Further for any $c \in \widetilde{S}_{k+1}$, we have $Z_{k}^{n} c=d \in \widetilde{S}_{k}$ : $d \neq 0$, for some nonnegative integer $n$. Then $e=c-(n !)-1 a^{n} d$ satisfies $Z_{k}^{n-1} e=f \in \widetilde{S}_{k}$. Hence by induction $\widetilde{S}_{k+1}=\widetilde{S}_{k}[a]$. Then $L_{k+1}=L_{k}(a)$, which proves the theorem.

Preserve the notation used in the proof of Theorem 2.3. For each $k=$ $1,2, \cdots, s+1 ; \lambda \in \operatorname{spec}(S)$ (resp. $\lambda \in \operatorname{spec}(T))$ set $S_{k}(\lambda)=S(\lambda) \cap S_{k}$ (resp. $\widetilde{S}_{k}(\lambda)=T(\lambda) \cap \widetilde{S}_{k}$ ). Recall that by construction $\left[Y_{i}, Z_{j}\right]=\mu_{i j} Z_{j}$ : $\mu_{i j} \in \mathrm{C}$. Let $\mu_{j}$ denote the $r$-tuple with entries $\mu_{i j}$. 
Lemma 2.4. For all $j, k=1,2, \cdots, s+1$,

(1) $Z_{j} S_{k}(\lambda) \subset S_{k}\left(\lambda+\mu_{j}\right)$ : for all $\lambda \in \operatorname{spec}(S)$,

(2) $Z_{j} \widetilde{S}_{k}(\lambda) \subset \widetilde{S}_{k}\left(\lambda+\mu_{j}\right)$ : for all $\lambda \in \operatorname{spec}(T)$,

(3) $S_{k}=\bigoplus_{\lambda \in \operatorname{spec}(s)} S_{k}(\lambda)$ (direct sum).

Proof. (1) and (2) are clear. (3) holds for $k=s+1$ since $S_{s+1}=S$ and $S$ is a direct sum of its eigensubspaces. Assume (3) holds for all $k>j$. To show it holds for $k=j$ recall that $S_{j} \subset S_{j+1}$ and apply (1).

Corollary 2.5. Suppose $S_{k+1} \neq S_{k}$ for some $k \in(1,2, \cdots, s)$. Then there exists $\lambda_{k} \in \operatorname{spec}(T)$ such that $\tilde{S}_{k+1}=\tilde{S}_{k}\left[a_{k}\right]$ with $a_{k} \in \tilde{S}_{k+1}\left(\lambda_{k}\right)$.

Proof. Apply Lemma 2.4 to the latter half of the proof of Theorem 2.3, noting that we may choose $a^{\prime} \in S_{k+1}(\lambda)$ for some $\lambda \in \operatorname{spec}(S)$.

The conclusion of Theorem 2.3 shows that we may write $K=$ $K_{0}\left(y_{1}, y_{2}, \cdots, y_{t}\right)$. Assume $t \leq \operatorname{dim} g$; let $\left\{T_{i}\right\}: i=1,2, \cdots, t$, be a subbasis of a basis for $g$ and denote by $B$ the matrix with entries $T_{i} y_{j}$. Recall that $g=g_{1} \oplus g_{2}$ and let $\left\{\dot{Y_{i}}\right\},\left\{Z_{j}\right\}$ be the bases for $g$ defined in the proof of Theorem 2.3. Assume $\left\{Y_{a}\right\}: \alpha=1,2, \cdots, \gamma,\left\{Z_{\mu}\right\}: \mu=1,2, \cdots, \rho$ to be subbases on which $B$ is nondegenerate. Further assume that

$$
Z_{k} \in\left\{Z_{\mu}\right\} \text { given } L_{k+1} \neq L_{k} \text {. }
$$

Following Theorem 2.3 introduce subsets $\left\{y_{a}^{\prime}\right\},\left\{y_{\mu}^{\prime \prime}\right\}$ of $y_{i}$ such that $L=K_{0}\left(y_{1}^{\prime}, y_{2}^{\prime}, \cdots, y_{\gamma^{\prime}}^{\prime}\right), K=L\left(y_{1}^{\prime \prime}, y_{2}^{\prime \prime}, \cdots, y_{\rho^{\prime}}^{\prime \prime}\right)$. Note that $\gamma+\rho=\gamma^{\prime}+$ $\rho^{\prime}=t$. By (2.3) $\rho^{\prime} \leq \rho$. Since $y_{a}^{\prime} \in L, B_{\mu a}=0$ for the given ranges of $\mu, \alpha$. Then $\operatorname{det} B \neq 0$, implies $\rho^{\prime} \geq \rho$. Hence $\rho^{\prime}=\rho$ and $\gamma^{\prime}=\gamma$. Then the $B_{\mu \nu}$ are the entries of a square matrix which is upper triangular, has ones on the diagonal, and its remaining entries lie in $\tilde{S}$ which was shown to be identical with $L\left[y_{1}^{\prime \prime}, y_{2}^{\prime \prime}, \ldots, y_{\rho}^{\prime \prime}\right]$. Since the $y_{\alpha}^{\prime} \in L$, it follows that $\operatorname{det} B \in L$. As was shown, the $y_{i}$ may be chosen as eigenfunctions of the elements of $g_{1}$. Then $B_{a i}=\lambda_{a i} y_{i}: \lambda_{a i} \in \mathbf{C}$. Let $B^{-1}$ denote the inverse of $B$. Then $B_{a}^{-1} \in L$, for all $\alpha, \beta$, and $B^{-1}$ defines a matrix which is upper triangular, has ones on the diagonal, and its remaining entries lie in $\widetilde{S}$. Finally $B_{\alpha \mu}^{-1}$ are polynomials in the $y_{\nu}^{\prime \prime}$ over, $L$ with no constant terms.

Given $a \in K$, then

$$
Z a=b \in \tilde{S} \text { for all } Z \in g_{2} \text { implies that } a \in \tilde{S} \text {. }
$$

Indeed by definition of $\tilde{\xi}$, there exists for each $Z \in g_{2}$ a positive integer $m$ such that $Z^{m} b=0$. But then $Z^{m+1} a=0$ which implies that $a \in \tilde{S}$. 
We remark that the above conclusions hold should $\mathrm{C}$ be replaced by an arbitrary algebraically closed commutative field of characteristic zero.

\section{Proof of conjecture.}

Theorem 3.1 [4, Proposition III.8, Theorem III.9]. Given g solvable, then

(1) there exist pairwise commutative elements $A_{\alpha} \in U g: \alpha=1,2, \cdots, s$, algebraically independent over $\mathbf{C}$. Set $A=\mathbf{C}\left[A_{1}, A_{2}, \cdots, A_{s}\right]$ and let $K$ denote its quotient field. The $[g, K] \subset K$, and $K$ is a maximal commutative subfield of $D g$;

(2) there exist $T_{i} \in g: i=1,2, \cdots, t$, algebraically independent over $K$ such that $\left(1, T_{1}, T_{2}, \cdots, T_{t}\right)$ is a basis for the extension $\tilde{g}$ of $g$ by $K$. Further $D g=D \widetilde{g}$ and $\left[T_{i}, A_{a}\right]=A_{i a} \in K$ where the matrix $A$ with entries $A_{i a}$ is of rank $t$ in $K$.

(3) $\operatorname{dim}_{C} C(D g) \leq s-t$ and $C(D g) \subset K$, where $C$ denotes centre.

Set $K_{0}=C(D g)$. Set $m=\operatorname{dim} g, n=1 / 2 \operatorname{dim} \Omega$, where $\Omega$ is an orbit of maximal dimension in the dual $g *$ of $g$ [1].

Theorem 3.2. Suppose $g$ is solvable and algebraic. Define $m, n$ as above. Then $D g$ is isomorphic to $D_{n, k}$ with $k=m-2 n$.

Proof. By [1] (cf. [9, Lemma 7]), $\operatorname{Dim}_{C} C(D g)=m-2 n=k$. Since $g$ is algebraic, (2.1) applies to the algebra $A$ defined in (1) above. Hence $2 s \leq 2 m-2 n=m+k$. Yet $m=\operatorname{dim} g=s+t$, where $t$ is defined in (2). Then by ${ }^{\cdot}(3), 2 s \geq m+k$. Hence $k=s-t$ and $n=t$. Further $t=\operatorname{deg} K-\operatorname{deg} K_{0}$.

Since $A \in U g$, the space $V$ generated by ad $g$ on $A$ is finite dimensional. Further the algebra $S$ generated by $V$, being a subalgebra of $U g$, has no zero divisors. By (1) its quotient field is precisely $K$ which is commutative. Since $g$ is solvable algebraic, it follows by Lemma 2.1 that ad $g$ considered as a subalgebra of $g l(V)$ is solvable and almost algebraic. Recalling (1), (3) above, Theorem 2.3 applies to show that $K_{0}\left(y_{1}, y_{2}, \cdots, y_{t}\right)$. Further by [10], there exist $z_{i} \in C(D g)$, such that $K_{0}=C\left(z_{1}, z_{2}, \cdots, z_{k}\right)$. Set

$$
B_{\alpha}= \begin{cases}y_{\alpha}: & \alpha=1,2, \cdots, t, \\ z_{a-t}: & \alpha=t+1, \cdots, s .\end{cases}
$$

Define $B_{i a}^{\prime}=\Sigma_{\beta} A_{i \beta}\left(\partial B_{a} / \partial A_{\beta}\right)$. Clearly $\operatorname{rank} B^{\prime}=\operatorname{rank} A=t$ in $K$ by (2) above. Further define a $t \times t$ matrix $B$ with entries

$$
B_{i j}=\left[T_{i}, y_{j}\right]=B_{i j}^{\prime}: \quad i, j=1,2, \cdots, t .
$$


Then $\operatorname{det} B \neq 0$, since $B_{i a}^{\prime}=0: a>t$.

Set $x_{j}=\Sigma_{i} B_{j i}^{-1} T_{i}$. By construction

$$
\left[x_{i}, y_{j}\right]=\delta_{i j} 1
$$

whereas

$$
\left[y_{i}, y_{j}\right]=0 \text {, }
$$

since $y_{i} \in K$. Note that (3.2) implies $\left(\operatorname{ad} x_{i}\right) u=\partial / \partial y_{i}(u)$ for all $u \in K$. Then by (3.2) and (3.3): $\left[\left[x_{i}, x_{j}\right], y_{k}\right]=0$ for all $i, j, k$. Hence

$$
\left[\left[x_{i}, x_{j}\right], A_{a}\right]=0
$$

for all $i, j=1,2, \cdots, t, a=1,2, \cdots, s$. Yet $x_{i} \in \tilde{g}$ so by the maximality of $K:\left[x_{i}, x_{j}\right] \in K$. Set $\left[x_{i}, x_{j}\right]=f_{i j}$. By antisymmetry, (3.2) and the Jacobi identity applied to $\left[\left[x_{i}, x_{j}\right], x_{k}\right]$ we obtain

$$
\begin{aligned}
f_{i j}+f_{j i} & =0, \\
\partial_{k} f_{i j}+\partial_{i} f_{j k}+\partial_{j} f_{k i} & =0,
\end{aligned}
$$

where $\partial_{k}$ denotes differentiation in $y_{k}$. The theorem is proved if we can show that there exist $g_{i} \in K$, such that

$$
f_{i j}=\partial_{i} g_{j}-\partial_{j} g_{i}
$$

for all $i, j$. For then replacing $x_{i}$ by $x_{i}-g_{i}$, we obtain

$$
\left[x_{i}, x_{j}\right]=0 \text {. }
$$

Then by (2) above the $x_{i}, y_{i}, z_{j}: i=1,2, \cdots, t, j=1,2, \cdots, k$, generate $D g$. Recalling that $z_{j} \in K_{0}$, it follows by (3.2), (3.3) and (3.7) that $D g$ is isomorphic to $D_{n, k}$ as required.

Let $A_{r}$ be the $r$ th generator of $K$ constructed in the recurrence procedure of $\left[4\right.$, Lemma III.4]. Set $K_{1}=\mathrm{C}, K_{r+1}=K_{r}\left(A_{r}\right)$. Let $\left\{X_{i}\right\}$ be some basis for $g$. The cobase $\left\{T_{i}\right\}$ is obtained as a subbasis of $\left\{X_{i}\right\}$ by eliminating at each step one $X_{i}$ for which $\alpha_{i} \neq 0$ in the relation: $A_{r}=\Sigma_{j} a_{j} X_{j}$ : $\alpha_{j} \in K_{r}$. With $g=g_{1} \oplus g_{2}$, choose the bases $\left\{Y_{i}\right\},\left\{Z_{j}\right\}$ for $g_{1}, g_{2}$ described in $\S 2$. Should $a_{i} \neq 0$, for some $Y_{i}$, eliminate the $Y_{i}$. Otherwise eliminate the $Z_{j}$ belonging to the smallest $j$ for which $a_{j} \neq 0$. Let $\left\{Y_{a}\right\},\left\{Z_{\mu}\right\}$ denote the resulting cobase. By construction if $Z_{k}$ is not in the cobase

$$
Z_{k}=\sum_{\mu>k} \alpha_{k \mu} Z_{\mu}+\beta_{k}: \quad \alpha_{k \mu}, \beta_{k} \in K .
$$


Suppose $L_{k+1} \neq L_{k}$. By Theorem 2.3, $L_{k+1}=L_{k}\left(a_{k}\right)$ where $\left[Z_{k}, a_{k}\right]=1$ and $a_{k} \in L_{k+1}$. This contradicts (3.8). Hence (2.3) holds for $\left\{Z_{\mu}\right\}$. Further the conclusion $\rho^{\prime}=\rho$ (established in the discussion following (2.3)) implies that

$$
Z_{k} \in\left\{Z_{\mu}\right\} \text { if and only if } L_{k+1} \neq L_{k}
$$

Recalling the choice of the cobase substitution from (3.8) gives

$$
\begin{gathered}
{\left[Y_{\alpha}, Y_{\beta}\right]=0, \quad\left[Y_{\alpha}, Z_{\mu}\right]=C_{\alpha \mu} Z_{\mu}: \quad C_{\alpha \mu} \in \mathbf{C},} \\
{\left[Z_{\mu}, Z_{\nu}\right]=\sum_{\lambda} \gamma_{\mu \nu}^{\lambda} Z+\Gamma_{\mu \nu}: \quad \gamma_{\mu \nu}^{\lambda}, \Gamma_{\mu \nu} \in K .}
\end{gathered}
$$

We show that $\Gamma_{\mu \nu} \in \widetilde{S}$. By (2.4) and the nilpotency of $g_{2}$ it suffices to show that $\gamma_{\mu \nu}^{\lambda} \in \widetilde{S}$. Now by (3.9), recalling the definition of the $a_{k}$, we have

$$
\gamma_{\mu \nu}^{\lambda}=\left[\left[Z_{\mu}, Z_{\nu}\right], a_{\lambda}\right]-\sum_{\sigma=1}^{\lambda-1} \gamma_{\mu \nu}^{\sigma}\left[Z_{\sigma}, a_{\lambda}\right] .
$$

Then, using (2.4), induction on $\lambda$ proves the assertion.

Construct the $y_{i}$ and the $x_{i}$ as above. Then the only contribution to $\left[x_{i}, x_{j}\right]$ comes from $\Gamma_{\mu \nu}$. Recalling (\$2) that $B_{i \mu}^{-1} \in \tilde{S}$ we obtain

$$
\left[x_{i}, x_{j}\right]=\sum_{\mu \nu} B_{i \mu}^{-1} B_{j \nu}^{-1} \Gamma_{\mu \nu} \in \tilde{S} .
$$

Set $x_{\alpha}^{\prime}=\Sigma_{i} B_{\alpha_{i}}^{-1} T_{i}, x_{\mu}^{\prime \prime}=\Sigma_{i} B_{\mu i}^{-1} T_{i}$. Since by (3.10), the $f_{i j}$ are polynomials in the $y_{\mu}^{\prime \prime}$ over $L$, we may integrate (3.5) in the $y_{\mu}^{\prime \prime}$ to obtain functions $g_{i} \in S$ such that the $\bar{x}_{i}=x_{i}-g_{i}$, satisfy

$$
\left[\bar{x}_{i}, \bar{x}_{\mu}\right]=0 \text {, }
$$

for all $i, \mu$. This integration is performed as follows. Assume $\rho \geq 1$ and identify $y_{\rho}^{\prime \prime}$ with $y_{t}$. Set $g_{t}=0$ and $g_{i}=\int_{0}^{y_{t}} f_{t i} d y_{t}$, for $1 \leq i<t$. By (3.10) $f_{t i}$ is polynomial in $y_{t}$ and so $g_{i} \in \tilde{S}$ for all $i$. Set $f_{i j}^{\prime}=f_{i j}-\left(\partial_{i} g_{j}-\partial_{j} g_{i}\right)$. Then $f_{i j}^{\prime} \in \widetilde{S}$ and satisfies (3.5). $f_{t i}^{\prime}=0$ by construction, so by $(3.5) f_{i t}^{\prime}=0$ and $\partial_{t} f_{i j}^{\prime}=0$ for all $i, j$. Then (3.11) obtains by successive integration in each $y_{\mu}^{\prime \prime}$. Note further that $g_{i}$ as polynomials in the $y_{\mu}^{\prime \prime}$ over $L$ are chosen to have no constant terms.

We show that the $\left[\bar{x}_{\alpha}, \bar{x}_{\beta}\right]$, as polynomials in the $y_{\mu}^{\prime \prime}$ over $L$, have no constant terms. This property was demonstrated in $\S 2$ for the $B_{a \mu}^{-1}$. It also holds for the $\left[Y_{\gamma}, g_{a}\right]$ by choice of the $g_{a}$ and because the $y_{\mu}^{\prime \prime}$ are eigen- 
vectors of ad $Y_{\gamma}$ where $\left(\operatorname{ad} Y_{\gamma}\right) L \subset L$ for all $\gamma$. It is then sufficient to observe that

$$
\left[\bar{x}_{\alpha}, \bar{x}_{\beta}\right]=\sum_{\mu \nu} B_{\alpha \mu}^{-1} B_{\beta \nu}^{-1} \Gamma_{\mu \nu}+\left[x_{\beta}, g_{\alpha}\right]-\left[x_{\alpha}, g_{\beta}\right],
$$

where

$$
\left[x_{\beta}, g_{a}\right]=\sum_{\gamma} B_{\beta \gamma}^{-1}\left[Y_{\gamma}, g_{\alpha}\right]+\sum_{\mu} B_{\beta \mu}^{-1}\left[Z_{\mu}, g_{a}\right] .
$$

Yet $\left[\bar{x}_{\mu},\left[\bar{x}_{\alpha}, \bar{x}_{\beta}\right]\right]=0$ by (3.11) and the Jacobi identity, so $\left[\bar{x}_{\alpha}, \bar{x}_{\beta}\right] \in L$ by (3.2). Hence $\left[\bar{x}_{a}, \bar{x}_{\beta}\right]=0$. Combined with (3.11) it follows that the required functions $g_{i}$ exist and the theorem is proved.

Remarks. The theorem evidently fails should $k-\operatorname{deg} K_{0}$ be an odd integer. Yet given $\operatorname{deg} K_{0}=k$, it is sufficient that $g$ be almost algebraic. In this connection see $[2, \$ 8]$ for examples. If $g$ is not almost algebraic the integration of (3.5) may fail in $K$. For example, let $e_{i j}$ denote the usual canonical basis in $\operatorname{Hom}\left(\mathrm{C}^{5}, \mathrm{C}^{5}\right)$. Set $a_{1}=e_{12}-e_{55}, a_{2}=e_{23}-e_{44}, y_{1}=$ $e_{15}, y_{2}=e_{14}, z=e_{13}$. Let $h \subset g l\left(C^{5}\right)$ be the Lie algebra spanned by these elements. In $h$ we have the bracket relations $\left[a_{1}, a_{2}\right]=z,\left[a_{1}, y_{1}\right]=y_{1}$, $\left[a_{2}, y_{2}\right]=y_{2}$ and all other brackets vanish. $h$ is evidently solvable, yet not almost algebraic. Set $K=\mathbf{C}\left(y_{1}, y_{2}, z\right)$. Then $K_{0}=\mathbf{C}(z), x_{1}=y_{1}^{-1} a_{1}$, $x_{2}=y_{2}^{-1} a_{2}$. Equations (3.1) and (3.2) hold; but $f_{12}=z / y_{1} y_{2}$ and (3.5) does not admit integration in $K$.

Finally given $g$ nilpotent, it is easy to see that the common divisor of $x_{i}, y_{i}, z_{j}$ lies in $C(U g)$, so we have incidentally proved [2, Lemma 9].

Acknowledgements. This paper was presented at a meeting on enveloping algebras of Lie algebras held at the Mathematisches Forschungsinstitut, Oberwolfach. Simultaneously two further independent and different proofs of the conjecture were given by J. C. McConnell [11] and W. Borho [12]. I should also like to thank the referee for some suggested improvements and corrections to the original manuscript.

\section{REFERENCES}

1. I.M. Gel'fand and A.A. Kirillov, Fields associated with enveloping algebras of Lie algebras, Dokl. Akad. Nauk SSSR 167 (1966), 503-505 = Soviet Math. Dokl. 7 (1966), 407-409. MR 33 \#4108.

2. - Sur les corps liés aux algèbres enveloppantes des algèbres de Lie, Inst. Hautes Études Sci. Publ. Math. No. 31 (1966), 5-19. MR 34 \#7731.

3. - Structure of the Lie field connected with a split semisimple Lie algebra, Funkcional. Anal. i Priložen. 3 (1969), no. 1, 7-26. (Russian) MR $39 \# 2827$. 
4. X. H. Nghiêm, Bull. Sci. Math. 96 (1972), 111.

5. A. Joseph, Symplectic structure in the enveloping algebra of a Lie algebra, Bull. Math. Soc. France (to appear).

6. N. Jacobson, Lie algebras, Interscience Tracts in Pure and Appl. Math., no. 10, Interscience, New York, 1962. MR 26 \#1345.

7. C. Chevalley, A new kind of relationship between matrices, Amer. J. Math. 65 (1943), 521-531. MR 5, 171.

8. —, Algebraic Lie algebras, Ann. of Math. (2) 48 (1947), 91-100. MR 8, 435 .

9. J. Dixmier, Sur les représentations unitaires des groupes de Lie nilpotents. II, Bull. Soc. Math. France 85 (1957), 325-388. MR 20 \# 1928.

10. P. Bernat, Sur le corps enveloppant d'une algèbre de Lie résoluble, C.R. Acad. Sci. Paris 258 (1964), 2713-2715. MR 28 \#4014.

11. J. C. McConnell, Representations of solvable Lie algebras and the Gelfand-Kirillov conjecture, School of Mathematics, Univ. of Leeds, 1973 (preprint). 12. W. Borho, P. Gabriel and R. Rentschler, Primideale in Einhüllenden auflösbarer Lie-Algebren, Lecture Notes in Math., vol.357, Springer-Verlag, Berlin, 1973 (Chap. II, §8). ISRAEL

DEPARTMENT OF PHYSICS AND ASTRONOMY, TEL-AVIV UNIVERSITY, RAMAT-AVIV,

Current address: I.H.E.S., 91 Bures sur Yvette, France 